\title{
Body Fluids in End-Stage Renal Disease: Statics and Dynamics
}

\author{
Jeroen P. Kooman Frank M. van der Sande \\ Maastricht University Medical Center, Maastricht, The Netherlands
}

\section{Keywords}

Fluid overload $\cdot$ Hemodialysis $\cdot$ Bioimpedance

spectroscopy · Relative blood volume - Absolute blood

volume Oxygen saturation

\begin{abstract}
Background: Abnormalities in fluid status in hemodialysis (HD) patients are highly prevalent and are related to adverse outcomes. Summary: The inherent discontinuity of the HD procedure in combination with an often compromised cardiovascular response is a major contributor to this phenomenon. In addition, systemic inflammation and endothelial dysfunction are related to extracellular fluid overload (FO). Underlying this relation may be factors such as hypoalbuminemia and an increased capillary permeability, leading to an altered fluid distribution between the blood volume (BV) and the interstitial fluid compartments, compromising fluid removal during dialysis. Indeed, whereas estimates of extracellular volume by bioimpedance spectroscopy are highly predictive of mortality, absolute BV assessed by the saline dilution technique was predictive of intra-dialytic morbidity. Changes in relative BV during $\mathrm{HD}$ are positively related to ultrafiltration rate (UFR) and, at least in some studies, nega-
\end{abstract}

tively to FO. High UFR is also related to changes in central venous oxygen saturation $\left(\mathrm{S}_{\mathrm{cv}} \mathrm{O}_{2}\right)$, a marker for tissue perfusion. On the one hand, high UFR and more pronounced declines in $\mathrm{S}_{\mathrm{cv}} \mathrm{O}_{2}$, but on the other hand, flat relative BV curves are also predictive of mortality; the relation between outcome which statics and dynamics of fluid status appears to be complex. Key Message: While technological developments enable the clinician to monitor statics and dynamics of fluid status and hemodynamics during HD in an accessible way, the role of technology-based interventions needs further study.

(c) 2018 The Author(s)

Published by S. Karger AG, Basel

\section{Introduction}

Despite advances in renal replacement techniques over the past decades, abnormalities in fluid balance are highly prevalent in patients with end-stage renal disease and consistently related to adverse outcome [1-4]. Achieving normovolemia is a major goal of dialysis therapy, but this is notoriously difficult. While the discontinuity of hemodialysis (HD), often in combination with insufficient sodium restriction leads to a signifi- 
cant fluid gain, the association of ultrafiltration (UF) with dialysis hypotension, which is in itself also related to adverse outcome [5], limits the amount of fluid, which can be removed during a relatively short period of time. Recent studies have substantially increased our understanding regarding the pathophysiology of abnormalities in fluid state and their relationship with outcome, as well as on the effects of HD on fluid dynamics. The aim of this short review is to discuss novel insights in the pathophysiology and monitoring of body fluid regulation in HD patients and the potential of technology-assisted treatment in optimizing fluid regulation.

\section{Fluid Volume Compartments}

In the conventional paradigm, total body water (TBW) is subdivided into an extra-cellular volume (ECV) and intra-cellular volume in which the ECV is further subdivided into interstitial fluid volume and blood volume (BV). Although this model is grossly oversimplified [6,7], it is still useful for clinical purposes. These body compartments can be estimated using tracer dilution methods, although this is impractical in a clinical setting. Recently, a 3-compartment model has been designed based on bioimpedance spectroscopy (BIS) in which an "overhydration" $(\mathrm{OH})$ compartment is defined on the assumptions of a fixed hydration of adipose and lean tissue mass [8]. This model has, even within narrow limits, proven validity in predicting outcome in HD patients [2]. Although abnormalities in ECV are predictive of outcome, changes in the intravascular volume are important for the hemodynamic response to dialysis therapy, which is also an important predictor outcome [5]. Intravascular volume can be expressed by plasma or BV, with tracer dilution techniques as the gold standard [9]. Recently, a method was developed based on the infusion of $240 \mathrm{~mL}$ of purified dialysate by which absolute $\mathrm{BV}$ is calculated from changes in relative BV [10], which can in turn be measured by changes in hematocrit or proteins by online monitoring [11]. Intravascular volume is a pivotal determinant of effective arterial BV, which is a virtual construct indicating organ perfusion. Nevertheless, effective arterial BV is also influenced by other mechanisms such as cardiac function and (micro)vascular tone $[6,12]$. Recently, studies monitoring central venous oxygen saturation $\left(\mathrm{S}_{\mathrm{cv}} \mathrm{O}_{2}\right)$ during dialysis in patients with a dialysis catheter, as a proxy for organ perfusion, have shown the potential of this approach [13].

\section{Non-Osmotic Sodium Storage}

According to the classic theorem, TBW compartments are subdivided into an extracellular and intracellular compartment with an isotonic distribution of fluid. Building upon older observations, Titze et al. [7] designed a new paradigm in which the skin attained a novel role in blood pressure regulation by nonosmotic sodium storage. Recently, Olde Engberink et al. [14] observed that after infusion of hypertonic saline in healthy volunteers, only $47 \%$ could be retrieved in the urine, suggesting substantial sodium buffering. Another study in patients with heart failure found an increase in glycosaminoglycans in the skin, especially in the more negatively charged (sulfated) subtype $[15,16]$.

Other authors also suggested that the endothelial surface layer (ESL) may also serve as a buffer for sodium [17]. Using a method in which total BV is compared with an ESL permeable tracer, the ESL, which is estimated to be around 1.5-1.7 l, but was found to be around 0.21 in patients with type I diabetes mellitus and microalbuminuria $[18,19]$. Combining the lines of thought regarding the potential sodium buffering roles of the ESL and the skin, it may be hypothesized that a reduction in buffering function of the endothelium allows sodium to enter the endothelial cell. This may increase vascular stiffness $[17,18]$. Further translocation of sodium into the skin can stimulate lymphangiogenesis through the activation of vascular endothelial growth factor and activate the immune system, most notably through the induction of a pro-inflammatory phenotype of macrophages as well as by stimulation of Th-17 cells [7].

The hemodynamic effects of tissue sodium storage in the HD population are likely important but not yet completely clear. It has been shown that using ${ }^{23} \mathrm{Na}$ MRI that HD is able to mobilize skin as well as muscle sodium [20], although the efficacy may be dependent upon local or systemic regulatory mechanisms, as evidenced by the inverse relation between skin sodium and vascular endothelial growth factor levels $[16,21]$. At first glance, the nonosmotic storage of sodium would seem to be a beneficial adaptation mechanism to buffer extremes in sodium intake. However, this may be offset by adverse effects such as the induction of proinflammatory effects $[7,17,18,22]$ and insulin resistance [23]. In addition, interstitial sodium stores may possibly induce vascular dysfunction [24]. Moreover, skin sodium stores were significantly related to left ventricular mass, and stronger so as compared to the relation between FO assessed by BIS and left ventricular mass [25]. At present, the concept of nonosmotic so- 
dium storage has yielded extremely interesting pathophysiological insights. Future research will likely clarify whether this concept will also lead to therapeutic interventions beyond the traditional paradigm of restricting sodium intake and attainment of normovolemia in the dialysis population.

\section{Abnormalities in Fluid Volume Distribution}

While BV and ECV are linearly related [26], the ratio between BV and ECV may still be dependent upon other factors, such as alterations in serum oncotic pressure and an increased capillary permeability [6]. The effect of hypoalbuminemia on body fluid distribution was shown by John et al. [9], who observed a comparable magnitude of the plasma volume compartment despite significantly higher ECV in peritoneal dialysis (PD) with serum albumin levels below the median as compared to patients with albumin levels above this level.

Recent evidence suggests that other factors beyond the decline in colloid osmotic pressure, which is partly compensated by an increase in globulins, are also responsible for the altered fluid distribution in hypoalbuminemic states such as nephrosis and inflammation [27]. Recently, the role of the ESL layer in the pathogenesis of abnormalities in body fluid distribution has been highlighted. Damage of the ESL, as evidenced by the shedding of glycocalix products and/or sidestream darkfield imaging, has been found in several disease states that are associated with increased permeability such as sepsis but was also observed in end-stage renal disease [28-30]. Although fluid loading may lead to ESL shedding by atrial natriuretic peptide [28], there is as yet no direct evidence for a relation between ESL damage and fluid overload (FO) in dialysis patients. However, we recently observed higher levels of the vascular cell adhesion molecule 1 in FO as compared to normovolemic dialysis patients, suggesting a relation between endothelial dysfunction and FO [31]. Also, in chronic kidney disease non-dialysis patients, angiopoietin 2, a factor that stimulates capillary permeability, was significantly related to FO [32].

\section{Non-Cardiovascular Factors on Abnormalities in Fluid Status}

Various studies have shown a relation between FO and inflammation $[2,31,33]$. The relation between FO and inflammation is likely complex and has been discussed in detail in a recent review paper [34]. Although it appears likely that an increased capillary permeability would play a major role in this phenomenon, the single study addressing this subject observed that capillary leakage, assessed by the Iodine-125 albumin method, was more strongly related to markers of platelet activation as compared to inflammatory markers in a cohort of PD patients [35].

In addition, a relation between malnutrition and $\mathrm{FO}$ was also observed [36]. In this respect, it should be noted that ECV/TBW ratio, which is also often used in the assessment of fluid status, may also be influenced by a reduction in body cell mass leading to a reduction in intra-cellular volume [37]. We also observed lower levels of lean tissue mass and serum albumin levels in patients with more advanced FO [2]. Conversely, FO was more pronounced in patients with lean tissue index levels $<10 \%$ and even more so in the concomitant presence of inflammation. Also chronic kidney disease patients with stages $3-5, \mathrm{FO}$, assessed by BIS, were inversely associated with serum albumin and lean tissue index [38].

The mechanisms of the relation between FO and malnutrition remain to be elucidated and may range from a lack of adjustment of dry weight in cases where a decline in fat or lean tissue mass goes undetected, the combined presence of inflammation and hypoalbuminemia leading to an alteration in fluid distribution or the presence of underlying illness which makes the patient more prone to hemodynamic instability making the achievement of dry weight more difficult [34]. It has also been suggested that malnutrition without renal failure itself leads to an increase in the ECV: weight ratio [39], but this may be also due to a change in the denominator. In the paper of Barac-Nieto et al., who assessed body composition in non-uremic malnourished patients in detail, $\mathrm{BV}$ and ECV were comparable in absolute terms between patients with severe and mild nutritional compromise, but their percentual ratios were higher due to the lower body weight [40].

Also, diabetes has an influence on body fluid distribution. Kopp et al. [20] observed that, despite comparable TBW assessed by BIS, ECV was higher in HD patients with type II diabetes mellitus as compared to non-diabetics, whereas intracellular volume was lower. Also, skin and muscle sodium content, assessed by ${ }^{23} \mathrm{Na}$ MRI, were higher in the diabetics. Of interest, ECV correlated with $\mathrm{HbA} 1 \mathrm{C}$ in the diabetics. Summarizing, FO may be part of a spectrum in which comorbidity, malnutrition and inflammation may be present, greatly 
contributing to the risk profile $[2,34]$. Therefore, it appears prudent to look in fluid-overloaded FO patients carefully for abnormalities in other risk factors, such as the nutritional and inflammatory domains.

\section{The Dry Weight Concept}

Dry weight is clinically defined as the body weight at which the patient remains normotensive without antihypertensive medication [41]. Patients in whom dry weight was reached according to this operational definition, using long dialysis hours in combination with meticulous sodium restriction have lower extracellular volume and better survival [41-43]. In general, dry weight is assumed to coincide with normalization of ECV, although substantial differences may be observed between clinical judgment and information obtained by additional technologies [44, 45]. Interestingly, in a cohort study in 639 PD patients, $28 \%$ of the population was fluid overload, defined as $\mathrm{OH}$ : ECV ratio $>7 \%$, in combination with a normal or low blood pressure [1].

When BIS is used to define normovolemia, in contemporary cohorts of both HD and PD patients, a substantial subset of patients remains fluid overloaded even in clinics where this technique is routinely applied [1-3]. The prevalence of FO, defined as a predialytic $\mathrm{OH}$ : ECV ratio above $15 \%$ or an absolute $\mathrm{OH}$ volume of $>2.51$ varied between 25 and $46 \%$ [3, 4], and 60-63\% when a cut-off limit of an absolute $\mathrm{OH}$ volume of $>1.1$ 1 or an $\mathrm{OH}$ : ECV ratio $>7 \%$ was used $[1,46]$. The question as to why it is so difficult to achieve normovolemia in daily clinical practice arises due to an imbalance between fluid and sodium intake, and the possibility to remove fluid adequately despite the discontinuity, which is an integral component of HD. This problem is likely augmented in patients with substantial comorbidity and/or cardiac dysfunction $[47,48]$. To make matters even more complex, pre-dialytic fluid depletion is associated with increased mortality as compared to patients with normovolemia, whereas post-dialytic fluid depletion was associated with a better outcome as compared to post-dialytic normovolemia [2]. These results suggest that the optimal limits for fluid status are quite narrow, which also likely necessitates close monitoring of fluid status during the HD procedure. The following sections will address the relevance of dynamics in fluid state in treatment monitoring and optimization.

\section{Dynamics of Fluid State During Dialysis}

One important parameter in the tolerance to UF is the ratio between fluid removal and refill of plasma volume from the interstitium, which can be estimated by measuring the change in relative BV or plasma volume during dialysis [49]. High UF volume is associated with increased mortality [50] and a more pronounced drop in RBV [51]. On the other hand, so-called flat RBV curves are associated with increased mortality and are assumed to be a reflection of FO $[26,52,53]$, which may be due to increased refill from the interstitium or a too low UF rate (UFR).

By using appropriate methodology like Critline ${ }^{\circledR}$ (Fresenius Medical Care, North America), relative BV changes are very easy to monitor in routine clinical practice. Some caveats with RBV monitoring should be mentioned, as it may underestimate the drop in absolute volume due to the translocation of blood with a relatively lower hematocrit from the microcirculation [11].

Interestingly, the relation between the decline in RBV and fluid status has been debated as Keane et al. [51] did observe a relation between the decline in RBV and UFR but not with FO assessed by BIS. Notably, some of the patients who displayed a "flat curve" were classified as fluid depleted by BIS. Also, vascular refilling rate was found to be independent from pre-dialytic volume status [26] and was not different between dialysis sessions accompanied by morbid events and those that were not [54]. Kron et al. [26] suggested that while the decline in relative BV may be influenced by volume status, refilling rate may be dependent upon UFR. Recently, RBV changes during dialysis were found to be related to outcome. A recent study showed that RBV between 93 and 96\% in the first hour and between 86 and $92 \%$ in the $3 \mathrm{~h}$ of dialysis had the most optimal association with outcome [55].

Another relevant parameter in the intra-dialytic hemodynamic response is the change in $\mathrm{S}_{\mathrm{cv}} \mathrm{O}_{2}$, which can also be measured using the Critline ${ }^{\circledR}$ device. A study by the Renal Research Institute showed that patients with the largest decline in $\mathrm{S}_{\mathrm{cv}} \mathrm{O}_{2}$ had the worst survival [13]. At present, the routine estimation of changes in tissue perfusion is only possible in patients with a central venous catheter. Alternative methods such as near-infrared spectroscopy have not yet been evaluated in dialysis patients [56]. Another study by the same group showed that changes in $\mathrm{S}_{\mathrm{cv}} \mathrm{O}_{2}$ are related to UF volume [57].

Therefore, there appears to be an interesting, and not yet completely solved interaction between different parameters reflecting or influencing intra-dialytic hemody- 
namics. On the one hand, FO, which may be associated with flattening RBV curves, is consistently related to mortality. On the other hand, high UFR, which is associated with adverse outcomes, is associated with steeper RBV curves, which are associated with better outcomes but also with a larger decline in $\mathrm{S}_{\mathrm{cv}} \mathrm{O}_{2}$, which is again associated with worsened outcomes. In addition, high UF volumes are not always associated with the worst outcomes, which were actually observed in a group with both FO and low UF volumes [58]. Most likely, significant comorbidity may have hampered fluid removal in this group of patients and may also be a contributing factor to the paradoxical associations discussed above.

\section{Can Technology Aid the Clinical Decision Process?}

In recent years, much published evidence has emerged regarding the role of technology assistance in monitoring fluid status and to a lesser degree, in dialysis treatment prescription. The added value of technology assistance should be regarded in terms of its contribution to the clinical decision process, which can vary from identification of patients at risk to technology-directed treatment leading to improvement in hard outcomes.

Especially BIS, most notably by using the 3 compartment model, has shown to be related to outcome in various studies $[3,59,60]$, with an optimal survival found to be within a quite narrow range (pre-dialytic $\mathrm{OH}$ between -1.1 and +1.1 L) [61]. Also, pre-dialytic fluid depletion was associated with outcome but interestingly only when accompanied by low pre-dialytic systolic blood pressure [46]. Lung comets assessed by ultrasound, which are a marker of regional fluid accumulation, are also predictive of outcome [62]. The relation between outcome, RBV and $\mathrm{S}_{\mathrm{cv}} \mathrm{O}_{2}$ changes has been discussed in a previous section.

Regarding the prediction of hemodynamic instability during dialysis, an estimation of intravascular fluid holds promise but should be tested in larger populations. In a pilot study, an absolute BV below $65 \mathrm{~mL} / \mathrm{kg}$ was found to predict intra-dialytic morbid events in 10 out of 12 patients [10]. Absolute BV was more predictive for intra-dialytic morbid events than pre-dialytic $\mathrm{OH}$ assessed by BIS [63]. Previously, it was also observed that vena cava diameter, which is also related to intravascular volume, predicted treatment tolerance in an intensive care setting [64].

Data regarding the effects of technology-assisted intervention are scarcer. Although one study showed a reduction in mortality with BIS-guided treatment [65], this did not reach significance in 2 subsequent meta-analyses. However, a reduction in pre-dialytic blood pressure was observed with the use of BIS, as well as a reduction in [39, 66] FO especially in patients without residual renal function. Enthusiasm for the use of relative BV as a tool for treatment prescription has subsided, since the results of the CLIMB study, which showed that the use of a protocol based on changes in relative BV, was actually associated with an increased mortality. However, it should be noted that the mortality in the control group was unexpectedly low, whereas the protocol used in the study was voluntary and not based on a strictly controlled prescription policy [67]. In the DRIP trial, patients with "flat" relative BV curves showed the greatest decline in interdialytic blood pressure upon reducing dry weight [68].

Regarding the use of absolute $\mathrm{BV}$ in the reduction of intra-dialytic morbid events, a small uncontrolled trial combining absolute BV and BIS resulted in a decline of intra-dialytic morbid events from 12.2 to $0.9 \%$ [62].

These recent data suggest that a possible strategy would be to use BIS or lung comets to identify patients at risk $[61,69]$, to use absolute BV (or vena cava ultrasound) to assess the risk of intra-dialytic morbid events, and (when applicable) $\mathrm{S}_{\mathrm{cv}} \mathrm{O}_{2}$ to monitor the response to dialysis treatment. Monitoring changes in RBV can additionally be used to see whether patients do not display a "flat curve" during UF, which may be an additional argument for FO [70], although given the recent evidence, the interpretation of relative $\mathrm{BV}$ curves as marker of fluid state deserves further study.

As an example, in patients who are fluid overloaded according to BIS, but in whom absolute BV is relatively low and the dialysis treatment is accompanied by substantial decrease in $\mathrm{S}_{\mathrm{cv}} \mathrm{O}_{2}$ and a major decline in relative $\mathrm{BV}$, a shift to more frequent and/or prolonged treatment appears indicated. Assessment of FO, absolute BV and relative $\mathrm{BV}$, as well as $\mathrm{S}_{\mathrm{cv}} \mathrm{O}_{2}$ necessitates only 2 devices and could be easily evaluated in clinical practice as techniques are simple and operator-independent. It should be acknowledged that this strategy is only based on theory and has not been formally evaluated.

\section{Conclusion}

Abnormalities in fluid status, most notably extracellular FO are associated with increased mortality. Despite the fact that there is no discussion regarding the importance of attaining normovolemia, it appears notoriously difficult to achieve in clinical practice. This is due to the 
difficulty in removing the fluid gain attributable to the intermittency of the treatment and the inappropriate adherence to a strict salt restricted diet in the short period of time allocated for the dialysis treatment. Easily applicable technology like BIS may aid in identifying patients with extracellular FO at risk for adverse outcomes, whereas measurements of absolute BV may predict the tolerance to dialysis treatment. Monitoring of RBV and where possible $\mathrm{S}_{\mathrm{cv}} \mathrm{O}_{2}$ during $\mathrm{HD}$ may predict the tolerance of the treatment and identify patients who would tolerate fluid removal without risk of a critical decline in organ perfusion. At present, the proven validity of technology-assisted treatment mainly lies in the identification of patients at risk, but its role in the actual prevention of dialysis-related complications should be addressed in future trials.

\section{References}

1 Van Biesen W, et al: Fluid status in peritoneal dialysis patients: the European Body Composition Monitoring (EuroBCM) study cohort. PLoS One 2011;6:e17148.

2 Dekker MJ, et al: Impact of fluid status and inflammation and their interaction on survival: a study in an international hemodialysis patient cohort. Kidney Int 2017;91:1214-1223.

3 Zoccali C, et al: Chronic Fluid Overload and Mortality in ESRD. J Am Soc Nephrol 2017; 28:2491-2497.

4 Wizemann V, et al: The mortality risk of overhydration in haemodialysis patients. Nephrol Dial Transplant 2009;24:1574-1579.

5 Flythe JE, et al: Association of mortality risk with various definitions of intradialytic hypotension. J Am Soc Nephrol 2015;26:724-734.

6 Roumelioti ME, et al: Fluid balance concepts in medicine: principles and practice. World J Nephrol 2018;7:1-28.

7 Wiig H, Luft FC, Titze JM: The interstitium conducts extrarenal storage of sodium and represents a third compartment essential for extracellular volume and blood pressure homeostasis. Acta Physiol (Oxf) 2018;222.

8 Chamney PW, et al: A whole-body model to distinguish excess fluid from the hydration of major body tissues. Am J Clin Nutr 2007;85: 80-89.

9 John B, et al: Plasma volume, albumin, and fluid status in peritoneal dialysis patients. Clin J Am Soc Nephrol 2010;5:1463-1470.

10 Kron S, et al: Determination of the critical absolute blood volume for intradialytic morbid events. Hemodial Int 2016;20:321-326.

11 Dasselaar JJ, van der Sande FM, Franssen CF: Critical evaluation of blood volume measurements during hemodialysis. Blood Purif 2012; 33:177-182.

12 Jacob M, Chappell D, Becker BF: Regulation of blood flow and volume exchange across the microcirculation. Crit Care 2016;20:319.

13 Chan L, et al: Intradialytic central venous oxygen saturation is associated with clinical outcomes in hemodialysis patients. Sci Rep 2017; 7:8581.

14 Olde Engberink RH, et al: Quantification of nonosmotic sodium storage capacity following acute hypertonic saline infusion in healthy individuals. Kidney Int 2017;91:738-745.
15 Nijst $\mathrm{P}$, et al: Dermal Interstitial alterations in patients with heart failure and reduced ejection fraction: a potential contributor to fluid accumulation? Circ Heart Fail 2018;11: e004763.

16 Wiig H: Regulation of fluid volume from the outside: a role of glycosaminoglycans in the skin interstitium? Circ Heart Fail 2018;11: e005135.

17 Wenstedt EF, Olde Engberink RH, Vogt L: Sodium handling by the blood vessel wall: critical for hypertension development. Hypertension 2018;71:990-996.

18 Olde Engberink RH, et al: Role of the vascular wall in sodium homeostasis and salt sensitivity. J Am Soc Nephrol 2015;26:777-783.

19 Nieuwdorp M, et al: Endothelial glycocalyx damage coincides with microalbuminuria in type 1 diabetes. Diabetes 2006;55:1127-1132.

20 Kopp C, et al: Elevated tissue sodium deposition in patients with type 2 diabetes on hemodialysis detected by ${ }^{23} \mathrm{Na}$ magnetic resonance imaging. Kidney Int 2018;93:1191-1197.

21 Dahlmann A, et al: Magnetic resonance-determined sodium removal from tissue stores in hemodialysis patients. Kidney Int 2015;87: 434-441.

22 Kleinewietfeld $\mathrm{M}$, et al: Sodium chloride drives autoimmune disease by the induction of pathogenic TH17 cells. Nature 2013;496: 518-522.

23 Deger SM, et al: Tissue sodium accumulation and peripheral insulin sensitivity in maintenance hemodialysis patients. J Cachexia Sarcopenia Muscle 2017;8:500-507.

24 Laffer CL, et al: Hemodynamics and salt-andwater balance link sodium storage and vascular dysfunction in salt-sensitive subjects. $\mathrm{Hy}$ pertension 2016;68:195-203.

25 Schneider MP, et al: Skin sodium concentration correlates with left ventricular hypertrophy in CKD. J Am Soc Nephrol 2017;28: $1867-1876$

26 Kron S, et al: Vascular refilling is independent of volume overload in hemodialysis with moderate ultrafiltration requirements. Hemodial Int 2016;20:484-491.

27 Bhave G, Neilson EG: Body fluid dynamics: back to the future. J Am Soc Nephrol 2011;22: 2166-2181.
28 Pillinger NL, Kam P: Endothelial glycocalyx: basic science and clinical implications. Anaesth Intensive Care 2017;45:295-307.

29 Cornelis T, et al: Effects of ultrapure hemodialysis and low molecular weight heparin on the endothelial surface layer. Blood Purif 2014;38:203-210.

30 Vlahu CA, et al: Damage of the endothelial glycocalyx in dialysis patients. J Am Soc Nephrol 2012;23:1900-1908.

31 Mitsides N, et al: Extracellular overhydration linked with endothelial dysfunction in the context of inflammation in haemodialysis dependent chronic kidney disease. PLoS One 2017;12:e0183281.

32 Tsai YC, et al: The interaction between fluid status and angiopoietin-2 in adverse renal outcomes of chronic kidney disease. PLoS One 2017;12:e173906.

33 Demirci MS, et al: Relations between malnutrition-inflammation-atherosclerosis and volume status. The usefulness of bioimpedance analysis in peritoneal dialysis patients. Nephrol Dial Transplant 2011;26:1708-1716.

34 Dekker MJE, et al: Fluid overload and inflammation axis. Blood Purif 2018;45:159-165.

$35 \mathrm{Yu} \mathrm{Z}$, et al: Hypoalbuminaemia, systemic albumin leak and endothelial dysfunction in peritoneal dialysis patients. Nephrol Dial Transplant 2012;27:4437-4445.

36 Cheng LT, Tang W, Wang T: Strong association between volume status and nutritional status in peritoneal dialysis patients. Am J Kidney Dis 2005;45:891-902.

37 Kim EJ, et al: Extracellular fluid/intracellular fluid volume ratio as a novel risk indicator for all-cause mortality and cardiovascular disease in hemodialysis patients. PLoS One 2017;12: $\mathrm{e} 0170272$.

38 Hung SC, et al: Volume overload correlates with cardiovascular risk factors in patients with chronic kidney disease. Kidney Int 2014; 85:703-709.

39 Tabinor M Davies SJ: The use of bioimpedance spectroscopy to guide fluid management in patients receiving dialysis. Curr Opin Nephrol Hypertens 2018;27:406-412.

40 Barac-Nieto $\mathrm{M}$, et al: Body composition in chronic undernutrition. Am J Clin Nutr 1978; 31:23-40. 
41 Charra B, et al: Clinical determination of dry body weight. Hemodial Int 2001;5:42-50.

42 Katzarski KS, et al: Fluid state and blood pressure control in patients treated with long and short haemodialysis. Nephrol Dial Transplant 1999;14:369-375.

43 Charra B, et al: Long $3 \times 8 \mathrm{hr}$ dialysis: a threedecade summary. J Nephrol 2003;16(suppl 7): S64-S69.

44 Alexiadis G, et al: Comparison of multiple fluid status assessment methods in patients on chronic hemodialysis. Int Urol Nephrol 2017; 49:525-532.

45 Vasko R, et al: Clinical judgment is the most important element in overhydration assessment of chronic hemodialysis patients. Clin Exp Nephrol 2013;17:563-568.

46 Dekker M, et al: Pre-dialysis fluid status, predialysis systolic blood pressure and outcome in prevalent haemodialysis patients: results of an international cohort study on behalf of the MONDO initiative. Nephrol Dial Transplant 2018, Epub ahead of print.

47 Kooman JP, et al: Hemodialysis: a model for extreme physiology in a vulnerable patient population. Semin Dial 2018;31:500-506.

48 van der Sande FM, et al: Novel insights into the pathogenesis and prevention of intradialytic hypotension. Blood Purif 2018;45:230235.

49 Barth C, et al: Characteristics of hypotensionprone haemodialysis patients: is there a critical relative blood volume? Nephrol Dial Transplant 2003;18:1353-1360.

50 Assimon MM, Flythe JE: Rapid ultrafiltration rates and outcomes among hemodialysis patients: re-examining the evidence base. Curr Opin Nephrol Hypertens 2015;24:525-530.
51 Keane DF, et al: Time to reconsider the role of relative blood volume monitoring for fluid management in hemodialysis. ASAIO J 2018, Epub ahead of print.

52 Lopot F: Clinical use of continuous blood volume monitoring. EDTNA ERCA J 1996;22: 7-11.

53 Agarwal R: Volume overload in dialysis: the elephant in the room, no one can see. Am J Nephrol 2013;38:75-77.

54 Kron S, et al: Vascular refilling is not reduced in dialysis sessions with morbid events. Blood Purif 2017;43:309-314.

55 Preciado P, Zhang H, Thijssen S, Kooman JP, van der Sande FM, Kotanko P: All-cause mortality in relation to changes in relative blood volume during hemodialysis. Nephrol Dial Transplant 2018, Epub ahead of print. https:// academic.oup.com/ndt/advance-article/ doi/10.1093/ndt/gfy286/5101401.

56 Chen YJ, et al: Cerebral desaturation in heart failure: potential prognostic value and physiologic basis. PLoS One 2018;13:e0196299.

57 Zhang H, et al: Association between intradialytic central venous oxygen saturation and ultrafiltration volume in chronic hemodialysis patients. Nephrol Dial Transplant 2017, Epub ahead of print.

58 Hecking M, et al: Greater fluid overload and lower interdialytic weight gain are independently associated with mortality in a large international hemodialysis population. Nephrol Dial Transplant 2018; 33:1832-1842.

59 Onofriescu M, et al: Overhydration, cardiac function and survival in hemodialysis patients. PLoS One 2015;10:e0135691.

60 Dekker MJE, Kooman JP: Fluid status assessment in hemodialysis patients and the association with outcome: review of recent literature. Curr Opin Nephrol Hypertens 2018;27: 188-193.

61 de Goeij MC, et al: Association of blood pressure with the start of renal replacement therapy in elderly compared with young patients receiving predialysis care. Am J Hypertens 2012;25:1175-1181.
62 Covic A, Siriopol D, Voroneanu L: Use of lung ultrasound for the assessment of volume status in CKD. Am J Kidney Dis 2018;71:412422

63 Kron S, et al: Adjustment of target weight based on absolute blood volume reduces the frequency of intradialytic morbid events. Hemodial Int 2018;22:254-260.

64 Chimot L, et al: Can we predict poor hemodynamic tolerance of intermittent hemodialysis with echocardiography in intensive care patients? J Ultrasound Med 2014;33: 2145-50

65 Onofriescu M, et al: Bioimpedance-guided fluid management in maintenance hemodialysis: a pilot randomized controlled trial. Am J Kidney Dis 2014;64:111-118.

66 Covic A, et al: Value of bioimpedance analysis estimated "dry weight" in maintenance dialysis patients: a systematic review and metaanalysis. Int Urol Nephrol 2017;49:22312245.

67 Reddan DN, et al: Intradialytic blood volume monitoring in ambulatory hemodialysis patients: a randomized trial. J Am Soc Nephrol 2005; 16:2162-2169.

68 Sinha AD, Light RP, Agarwal R: Relative plasma volume monitoring during hemodialysis AIDS the assessment of dry weight. Hypertension 2010;55:305-311.

69 Tabinor M, et al: Bioimpedance-defined overhydration predicts survival in end stage kidney failure (ESKF): systematic review and subgroup meta-analysis. Sci Rep 2018;8:4441.

70 Agarwal R: Relative plasma volume monitoring for identifying volume-sensitive and -resistant hypertension. Semin Dial 2010;23: 462-465. 